\title{
Reflets
}

Revue d'intervention sociale et communautaire

\section{Agir! Pour masanté : une interven- tion harmonisant services médicaux et services communautaires pour les Franco-Manitobains souffrant de dépression}

Danielle de Moissac, Monique Bohémier, Chelsea Herdman, Margaux Roch-Gagné, Julie Paillé, Christine Johnston, Michelle Arpin-Molinski, Georgette Dupuis, Mélanie Saint-Hilaire et Liliane Prairie

Volume 18, numéro 1, printemps 2012

URI : https://id.erudit.org/iderudit/1012336ar

DOI : https://doi.org/10.7202/1012336ar

Aller au sommaire du numéro

Éditeur(s)

Reflets, Revue d'intervention sociale et communautaire

ISSN

1203-4576 (imprimé)

1712-8498 (numérique)

Découvrir la revue

Citer cet article

de Moissac, D., Bohémier, M., Herdman, C., Roch-Gagné, M., Paillé, J., Johnston, C., Arpin-Molinski, M., Dupuis, G., Saint-Hilaire, M. \& Prairie, L. (2012). Agir! Pour masanté : une interven- tion harmonisant services médicaux et services communautaires pour les Franco-Manitobains souffrant de dépression. Reflets, 18(1), 155-163. https://doi.org/10.7202/1012336ar 


\section{Agir! Pour ma santé : une interven- tion harmonisant services médicaux et services communautaires pour les Franco-Manitobains souffrant de dépression}

Danielle de Moissac', Monique Bohémier, Chelsea Herdman, Margaux Roch-Gagné, Julie Paillé

Université de Saint-Boniface

Christine Johnston, Michelle Arpin-Molinski, Georgette Dupuis, Mélanie Saint-Hilaire et Liliane Prairie

Centre de santé Saint-Boniface

Le Centre de santé Saint-Boniface, un centre de soins primaires situé dans un quartier francophone de Winnipeg au Manitoba, est reconnu pour sa prestation de services de santé innovateurs qui favorisent la santé et le bien-être de sa communauté (Centre de santé, 2008). Il a pour mission de stimuler et de soutenir les francophones de la ville de Winnipeg et, plus particulièrement, la population de Saint-Boniface, dans la prise en charge de leur santé et de leur bien-être. Le Centre de santé collabore avec la communauté afin de promouvoir la santé et de prévenir, de traiter et de gérer les problèmes de santé. On peut y recevoir des soins médicaux, des services d'infirmières praticiennes et d'infirmières de santé primaire, ainsi que des services de nutrition, d'activité physique et de santé mentale. De plus, le Centre abrite le service d'Info-Santé, un service d'appel téléphonique pour une évaluation professionnelle des besoins de santé. Le Centre mise beaucoup sur 
le développement communautaire en cernant les préoccupations des membres de la communauté, en faisant la promotion des ressources et des services disponibles et en assurant un appui aux groupes qui veulent améliorer la santé de leur communauté (Centre de santé, 2008).

En 2008-2009, le Centre de santé Saint-Boniface a mis sur pied un projet pilote, le programme Agir! Pour ma santé (SaintHilaire, 2009), qui visait à offrir un soutien personnalisé aux adultes atteints de dépression légère ou modérée et qui préconisait l'utilisation de soins complémentaires de pair avec les soins médicaux traditionnels. Le programme avait pour objectifs de démontrer (Saint-Hilaire, 2009, p.7) :

1. que des services complémentaires, et particulièrement l'activité physique, peuvent diminuer le niveau de dépression et augmenter la qualité de vie des francophones souffrant de dépression légère à modérée;

2. que l'approche selon les principes du coaching-santé, où la promotion de la santé se fait grâce à une relation personnalisée de soutien, favorise à long terme la prise en charge de la santé par le client;

3. qu'un partenariat entre les services médicaux et les services communautaires peut être un moyen d'augmenter l'accès en français à des services de santé sécuritaires et de qualité.

Les composantes du programme Agir! Pour ma santé comprennent la nutrition, l'activité physique, la relaxation et la bibliothérapie. Lors de l'évaluation de ce projet pilote, les huit clients qui y ont participé ont perçu une réduction de leur niveau de dépression et une augmentation de leur qualité de vie. De plus, une réduction dans le nombre de visites auprès des médecins omnipraticiens a été notée. Les participants du projet, les intervenants en santé et les organismes communautaires ont apprécié le programme dans son ensemble, car il encourageait la prise en charge de la santé par le client, qui se rendait compte des avantages d'adopter de saines habitudes de vie. Pour le professionnel de la santé, l'occasion d'explorer un moyen alternatif d'aider son client, tout en mettant en valeur les services des autres membres de l'équipe 
multidisciplinaire, a été une expérience positive. Comme l'a dit un des partenaires communautaires : « La collaboration est à la base de tout [...] Ce projet renforce la nécessité de travailler en collaboration" (Saint-Hilaire, 2009, p. 44).

C'est pour plusieurs raisons que le Centre de santé SaintBoniface a choisi de mener ce projet pilote et d'assurer la pérennité de ce programme axé sur la dépression. Le Centre reconnaît d'abord l'importance d'une bonne santé mentale dans toutes les facettes de la vie, tant physiques, qu'émotionnelles ou sociales. Puis, un sentiment de frustration à l'égard de l'incapacité d'aider les clients souffrant d'une dépression légère ou modérée a motivé les intervenants à s'informer sur les meilleures pratiques citées dans la littérature et à s'inspirer d'un programme provenant d'un centre de santé américain qui fait la promotion de la prise en charge de la santé par le client lui-même, avec l'appui de plusieurs intervenants dans un contexte communautaire. Enfin, une pénurie de professionnels en santé mentale pouvant offrir des services en français se fait sentir au Manitoba, rendant difficilement accessible la psychothérapie pour les clients qui souhaitent recevoir ce service en français (de Moissac, et collab., 2011).

Pour bien comprendre le programme, il faut d'abord prendre connaissance de ce qu'est le trouble visé. Selon l'Association canadienne pour la santé mentale (2009), la dépression est la plus soignable des maladies mentales, mais moins du tiers des personnes qui souffrent de dépression grave demandent de l'aide (Association médicale canadienne, 2008). En effet, selon Santé Canada (2009), environ $11 \%$ des Canadiens et $16 \%$ des Canadiennes feront une dépression majeure au cours de leur vie. Une dépression est caractérisée par un état de tristesse et de désespoir inhabituel qui persiste plus de deux semaines et qui a un effet négatif sur le rendement professionnel, scolaire ou social (Centre de toxicomanie et de santé mentale, 2009). Elle peut aussi avoir des effets physiques, tels une perte d'appétit, une modification des habitudes de sommeil, des maux de tête et des troubles digestifs. La dépression peut avoir de multiples impacts. Par exemple, elle peut diminuer la qualité de vie, entraîner des difficultés relationnelles et engendrer certaines maladies chroniques, comme le diabète ou 
les maladies du cœur (Santé Canada, 2009). La dépression peut même mener au suicide. Généralement, la personne déprimée se replie sur elle-même et évite les contacts sociaux, ce qui a un impact sur ses relations professionnelles, familiales et sociales (Granger, 2010).

Le traitement médical de première ligne pour la dépression est l'ordonnance d'antidépresseurs et la psychothérapie (Cuijpers, et collab., 2009). La psychothérapie cognitive-comportementale est une approche qui tente de renseigner le patient et de lui offrir des outils ou des moyens pour mieux gérer sa santé mentale (Hofmann et Smits, 2008). Une telle thérapie peut inclure des techniques de relaxation, une réflexion sur la motivation, ou des techniques pour mieux dormir. Les études démontrant les bénéfices de l'exercice dans le traitement de la dépression sont aussi nombreuses (Mead, et collab., 2009; Lawlor et Hopker, 2001; Craft et Perma, 2004), ainsi que les études faisant un lien entre une bonne alimentation et la santé mentale (Akbaraly, et collab., 2009; Milte, et collab., 2009). Une approche globale, incorporant toutes ces facettes, augmente les chances de maintenir une bonne santé mentale. Le défi à surmonter est de trouver l'approche qui incitera les gens à prendre en charge leur santé. Selon les meilleures pratiques proposées dans la littérature, l'approche préconisée suit les principes du coaching-santé, où la promotion de la santé se fait au moyen d'une relation personnalisée de soutien (Palmer, 2003; Hayes, et collab., 2008), offerte au niveau des soins primaires (Cuijpers et collab., 2009; McGrady et collab., 2009; Conradi et collab., 2007; Forsyth et collab., 2009). Le client est mis au courant des ressources disponibles dans sa communauté et est encouragé et appuyé dans sa démarche. Il amorce ainsi des changements dans son comportement qui font qu'il demeure motivé à prendre en charge sa santé à long terme. Des études d'analyse coûts-bénéfices montrent que de tels soins sont plus efficaces (Sava et collab., 2009; Barrett et collab., 2005), avec une réduction du nombre de visites chez le médecin.

Le programme Agir! Pour ma santé est maintenant en vigueur depuis janvier 2010, avec une composante recherche financée par le Consortium national de formation en santé 2 . Le programme 
est constitué d'une intervention de douze semaines comprenant un suivi en activité physique obligatoire, des consultations nutritionnelles, de la psychothérapie au besoin et d'une session d'information sur les techniques de relaxation et la bibliothérapie. Une évaluation du niveau de dépression, de la qualité de vie, de l'activité physique et de la nutrition est effectuée avant et après l'intervention. Une évaluation à long terme, soit trois mois après l'intervention, permet de déterminer si le client a su maintenir un style de vie plus actif et une meilleure santé mentale.

Ce programme n'est viable qu'avec l'appui des ressources communautaires locales. Le Centre de santé Saint-Boniface est situé à proximité de l'Université de Saint-Boniface, où est logé le Sportex, un centre de conditionnement physique bilingue. On y retrouve un équipement varié pour l'exercice aérobique et pour la musculation; on y offre aussi des sessions de yoga. Le Sportex constitue un appui important au programme Agir! Pour ma santé, car il offre aux participants un lieu sécuritaire et familier dans lequel ils se sentent à l'aise de s'adonner à de nouvelles activités physiques et de s'exercer sur divers appareils. C'est un endroit très accueillant, particulièrement pour les participants qui n'ont pas l'habitude de fréquenter un centre de conditionnement physique et pour qui toute initiative représente un défi.

La Bibliothèque de Saint-Boniface est également un partenaire communautaire qui offre un appui en bibliothérapie. La bibliothèque propose des livres portant sur la dépression et sur les bienfaits de l'activité physique et de l'alimentation saine sur le maintien d'une bonne santé mentale, tel que recommandé par les professionnels du Centre de santé. Les participants peuvent donc profiter de cette ressource communautaire pour s'informer sur ce qu'est la dépression et les façons d'y faire face.

Le recrutement de clients qui répondent aux critères d'inclusion et qui s'intéressent à une prise en charge personnelle de leur santé est particulièrement difficile en santé mentale. Près de 100 clients potentiels ont été évalués pour leur niveau de dépression, qui devait être de léger à modéré. Jusqu'à présent, 42 clients ont répondu aux critères, ont montré un intérêt pour le programme et ont accepté d'y participer. Plus de $80 \%$ des 
participants sont des femmes âgées de 22 à 73 ans, l'âge moyen étant de 43 ans. Six hommes ont été identifiés, âgés de 23 à 65 ans, l'âge moyen étant de 44 ans. Les participants ont été divisés de façon aléatoire en deux groupes, soit le groupe contrôle, qui ne fait pas l'objet d'intervention, mais qui procède aux diverses mesures d'évaluation au début et après trois mois d'attente, et le groupe expérimental, qui fait l'objet d'une intervention de 12 semaines. Neuf participants ont fait partie du groupe contrôle, 13 participants ont terminé le programme et neuf participants ont accepté de se faire évaluer trois mois après la fin du programme.

Les résultats préliminaires sont très révélateurs. Une évaluation de l'activité physique montre une augmentation significative dans le nombre de minutes consacrées à l'activité physique par semaine, ainsi qu'une augmentation dans l'intensité de l'activité physique. Le niveau d'activité pendant le programme ne se maintient pas trois mois après la fin du programme, mais une augmentation importante se voit en comparaison avec l'activité physique pratiquée avant la participation au programme. L'évaluation du niveau de dépression avant et après le programme montre qu'une réduction dans le niveau de dépression - d'un score moyen de 24 , qui indique une dépression modérée, à un niveau où les participants ont des hauts et des bas considérés comme normaux, avec un score moyen de 10 - est perçue chez tous les participants, contrairement à aucune réduction significative dans le groupe contrôle. De plus, cette tendance se maintient pour une période de trois mois après la fin du programme. La qualité de vie semble être rehaussée chez les participants qui ont pu profiter du programme, du fait qu'ils sont plus actifs, ce qui influence leur sentiment de bien-être et de santé générale. Encore, cette tendance se maintient et même augmente trois mois après la fin du programme.

Les participants du groupe expérimental ont évalué leur niveau de satisfaction par rapport au programme. Bien qu'ils aient participé au programme principalement sous la recommandation de leur médecin, plusieurs participants souhaitaient un appui et des conseils individualisés afin de devenir plus actifs et être en meilleure santé. Ce qui ressort chez tous est le désir de se prendre en main, de trouver une façon d'être en meilleure santé 
physique et mentale. Le besoin d'un appui pour les motiver et les soutenir dans leur démarche vers une meilleure santé était important. Les éléments du programme qui ont été les plus appréciés par les participants ont été le soutien offert par la coach en activité physique et l'environnement favorable du Sportex. Tous les participants ont grandement apprécié la coach en activité physique, comme le décrit ce participant : "J'ai beaucoup aimé travailler avec Christine, elle était vraiment upbeat, puis ça. [...] J'avais hâte d'y aller ». Le programme a permis aux participants de faire des choses qu'ils n'auraient peut-être pas osé faire d'euxmêmes, mais grâce à l'appui des intervenants et des ressources communautaires, ils ont trouvé des occasions d'être plus actifs. Ces changements s'accompagnent d'un sentiment de réussite, de fierté, d'avoir surmonté un défi. Par exemple, une participante qui prend son vélo pour se rendre au travail nous a dit : «Ça, c'est quelque chose que, pour moi, c'est un gros chef d'œuvre, bien, un accomplissement ». D'autres reconnaissent que ce n'est qu'un début, qu'ils doivent continuer à être actifs et à bien manger pour maintenir une bonne santé physique et mentale.

Plusieurs participants ont noté des changements quant à leurs habitudes nutritionnelles. Certains mangent de façon plus régulière, consomment moins de boissons sucrées ou adoptent tout simplement une perspective positive face aux habitudes, comme le décrit cette participante :

«Me permettre de jouir de la nourriture que moi j'aime et pas toujours magasiner avec mon fils en tête; mais de mettre de l'importance sur ce que moi j'aime manger et puis de pas m'inquiéter trop de mon poids et de pas m'inquiéter trop de ce que les personnes pensent quand ils me voient manger. »

En conclusion, le succès du programme Agir! Pour ma santé, qui encourage les personnes atteintes de dépression légère ou modérée à prendre en charge leur santé, provient de la qualité du service offert par les intervenants en santé primaire et la collaboration entre le Centre de santé Saint-Boniface et ses partenaires 
communautaires. En incorporant des services complémentaires aux services médicaux et en travaillant en équipe interdisciplinaire, on permet aux clients d'avoir accès à une thérapie complémentaire, ce qui augmente leur qualité de vie et leur santé physique et mentale.

\section{Notes}

1 Auteure principale : Danielle de Moissac. Elle peut être jointe à l'Université de Saint-Boniface, 200, av. de la Cathédrale, Winnipeg, MB, R2H 0H7. Téléphone : 204-237-1818 poste 348. Télécopieur : 204-237-3240.

2 Cette étude a été rendue possible grâce à l'appui financier du Consortium national de formation en santé et de Santé Canada.

\section{Bibliographie}

AKBARALY, Tasnime, et collab. (2009). «Dietary pattern and depressive symptoms in middle age ", Br J Psychiatry, Vol. 195, No 5, p. 408-413.

ASSOCIATION CANADIENNE POUR LA SANTÉ MENTALE (2009). «La dépression », réf. du 27 novembre 2009, http://www.cmha.ca/bins/content_page.asp?cid=3-86-87\&lang=2

ASSOCIATION MÉDICALE CANADIENNE (2008). "Des chiffres alarmants sur la dépression ", Le magazine santé canadienne, juillet-août.

BARRETT, Barbara, Sarah BYFORD et Martin KNAPP (2005). "Evidence of cost-effective treatments for depression: A systematic review ", Journal of Affective Disorders, Vol. 84, p. 1-13.

CENTRE DE SANTÉ SAINT-BONIFACE (2008). "A propos du Centre de santé », réf. du 15 novembre 2011, http://www.centredesante.mb.ca/aboutus.php.

CENTRE DE TOXICOMANIE ET DE SANTÉ MENTALE (2009). « La dépression : Guide d'information ", réf. du 14 novembre 2011, http://www.camh.net/fr/About_Addiction_Mental_Health/Mental_Health_Information/ Depressive_Illness/depressive_ill_understanding_fr.html.

CONRADI, Henk Jan, et collab. (2007). «Enhanced treatment for depression in primary care: longterm outcomes of a psycho-educational prevention program alone and enriched with psychiatric consultation or cognitive behavioral therapy ", Psychol Med, Vol. 37, N 6, p. 849-862.

CRAFT, Lynette, et Frank PERMA (2004). «The Benefits of Exercise for the Clinically Depressed », Prim Care Companion J Clin Psychiatry, Vol. 6, № 3, p. 104-111.

CUIJPERS, Pim, et collab. (2009). «Psychological treatment of depression in primary care: a metaanalysis », Br J Gen Pract,Vol. 59, № 559, p. 51-60.

CUIJPERS, Pim, et collab. (2009). «Adding psychotherapy to pharmacotherapy in the treatment of depressive disorders in adults: a meta-analysis ", J Clin Psychiatry, Vol. 70, № 9, p. 1219-1229.

DE MOISSAC, Danielle, et collab. (2011). «Disponibilité et accessibilité des services de santé en français au Manitoba », Winnipeg, Université de Saint-Boniface. 
FORSYTH, Adrienne, Frank DEANE et Peter WILLIAMS (2009). "Dietitians and exercise physiologists in primary care: lifestyle interventions for patients with depression and/or anxiety ", J Allied Health, Vol. 38, $\mathrm{N}^{\circ}$ 2, p. 63-68.

GRANGER, Bernard (2010). "Le trou noir de la dépression », Les Grands Dossiers Des Sciences Humaines, Vol. 20, p. 26-27.

HAYES, Eileen, et collab (2008). "Alliance not compliance: coaching strategies to improve type 2 diabetes outcomes ", J Am Acad Nurse Pract,Vol. 20, № 3, p. 155-162.

HOFMANN, Stephan, et Jasper SMITS. (2008). "Cognitive-behavioral therapy for adult anxiety disorders: a meta-analysis of randomized placebo-controlled trials ", J Clin Psychiatry, Vol. 69, No 4, p. 621-632.

LAWLOR, Debbie, et Stephen HOPKER (2001). «The effectiveness of exercise as an intervention in the management of depression: systematic review and meta-regression analysis of randomized controlled trials ", British Medical Journal,Vol. 322, p. 1-8.

MCGRADY, Angele, Julie BRENNAN et Denis LYNCH (2009). «Effects of wellness programs in family medicine ", Appl Psychophysiol Biofeedback, Vol. 34, No 2, p. 121-126.

MEAD, Gillian, et collab. (2009). "Exercise for depression ", Cochrane Database Syst Rev, Vol. 8, № 3, CD004366.

MILTE, Catherine, Natalie SINN et Peter HOWE (2009). «Polyunsaturated fatty acid status in attention deficit hyperactivity disorder, depression, and Alzheimer's disease: towards an omega-3 index for mental health? ", Nutr Rev, Vol. 67, No 10, p. 573-590.

PALMER, Steven (2003). " Health coaching to facilitate the promotion of healthy behaviour and achievement of health-related goals ", International Journal of Health Promotion and Education,Vol. 41, No 3, p. 91-93.

SAINT-HILAIRE, Micheline (2009). "Rapport d'évaluation du projet Agir! Pour ma santé dans le cadre de l'initiative du Centre de santé Saint-Boniface — La santé et le mieux-être : Les services médicaux en harmonie avec les services communautaires ", Winnipeg, Centre de santé Saint-Boniface.

SANTÉ CANADA (2009). «Votre santé et vous - la dépression », réf. du 14 novembre 2011, http:// www.hc-sc.gc.ca/hl-vs/alt_formats/pacrb-dgapcr/pdf/iyh-vsv/diseases-maladies/ depressionfra.pdf.

SAVA, Florin, et collab. (2009). " Cost-Effectiveness and Cost-Utility of Cognitive Therapy, Rational Emotive Behavioral Therapy, and Fluoxetine (Prozac) in Treating Depression: A Randomized Clinical Trial ", Journal of Clinical Psychology, Vol. 65, p. 36-52.

Cet article est dédié à Julie Paillé qui, par son énergie positive et sa joie de vivre, nous rappelle de vivre pleinement chaque moment et d'apprécier les petits plaisirs que nous offre la vie. 